\title{
Fronts from complex two-dimensional dispersal kernels: Theory and application to Reid's paradox
}

\author{
Joaquim Fort ${ }^{\text {a) }}$ \\ Department de Física, Universitat de Girona, 17071 Girona, Catalonia, Spain
}

(Received 26 October 2006; accepted 18 March 2007; published online 15 May 2007)

\begin{abstract}
Bimodal dispersal probability distributions with characteristic distances differing by several orders of magnitude have been derived and favorably compared to observations by Nathan et al. [Nature (London) 418, 409 (2002)]. For such bimodal kernels, we show that two-dimensional molecular dynamics computer simulations are unable to yield accurate front speeds. Analytically, the usual continuous-space random walks (CSRWs) are applied to two dimensions. We also introduce discrete-space random walks and use them to check the CSRW results (because of the inefficiency of the numerical simulations). The physical results reported are shown to predict front speeds high enough to possibly explain Reid's paradox of rapid tree migration. We also show that, for a time-ordered evolution equation, fronts are always slower in two dimensions than in one dimension and that this difference is important both for unimodal and for bimodal kernels. () 2007 American Institute of Physics. [DOI: 10.1063/1.2733631]
\end{abstract}

\section{INTRODUCTION}

Reaction-diffusion phenomena are observed in many applied biophysics systems, e.g., tumor growth, ${ }^{1}$ patterning in microchannel flows, ${ }^{2}$ nanobiosensors, ${ }^{3}$ etc. However, the diffusion (or Laplacian) approximation does not hold in some cases-not only in matter transport but also in heat conduction $^{4}$ and radiative transfer. ${ }^{5,6}$ Indeed, classical diffusion (Fick's law) is well known to break down in some relevant phenomena. One example is time-delayed diffusion due to the rest time of particles (or individuals) between successive jumps ${ }^{8,9}$ (this delay can be the same for all jumps $^{10-12}$ or there may be several possible delays ${ }^{13-15}$ ). A second case is sometimes called reaction dispersal. Broadly speaking, reaction dispersal refers to dispersal probability distributions (kernels) which do not vanish for very long jump distances (compared to the front width). This leads to the break down of the diffusion (or second-order) approximation. Reaction dispersal has been widely used in modeling population invasions. ${ }^{16-19}$ An especially important subcase is that of kernels with several components with characteristic distances differing by several orders of magnitude. It has been long suspected that such kernels may explain a very important, unsolved biophysical problem, namely, the fact that the observed speeds of forest postglacial recolonization fronts are much faster than those predicted by single-kernel reaction-dispersal models. This disagreement is called Reid's paradox. ${ }^{18}$ Many authors have shown that hypothetical longdistance dispersal (LDD) events could solve Reid's paradox ${ }^{18}$ but all previous approaches are based on kernels fitted to short-distance data, with purely hypothetical LDD events ${ }^{18,19}$ [and almost always using one-dimensional (1D) models]. However, Nathan and co-workers have derived and tested very interesting kernels with two components: a shortdistance component (of the order of $10 \mathrm{~m}$ ) and a second,

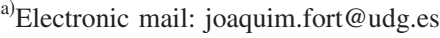

very rare, LDD component (covering distances of $10^{3}-10^{4} \mathrm{~m}$ but observed for only about $0.2 \%$ of seeds released from the parent tree). They derived such bimodal kernels by means of fluid dynamics simulations of atmospheric transport including turbulent-uplifting events that had been previously neglected. They also checked their new kernels by comparing predicted vertical deposition patterns and uplifting probabilities to observed data. ${ }^{7,20,21}$ This opens the possibility to explain Reid's paradox using complex dispersal kernels which are not hypothetical but derived from physical principles. Below we derive the appropriate front speed formulas for such complex kernels. We also show that the predicted front speeds are about $10^{2}-10^{3} \mathrm{~m} / \mathrm{yr}$ (which are two orders of magnitude higher than those obtained neglecting the LDD component). This could eventually solve Reid's paradox.

\section{EVOLUTION EQUATION}

Many two-dimensional (2D) reaction-dispersal models are based on the equation ${ }^{8-12,22}$

$$
\begin{aligned}
p(x, y, t+T)= & \int_{-\infty}^{+\infty} \int_{-\infty}^{+\infty} p\left(x+\Delta_{x}, y+\Delta_{y}, t\right) \\
& \times \phi\left(\Delta_{x}, \Delta_{y}\right) d \Delta_{x} d \Delta_{y}+R[p(x, y, t)],
\end{aligned}
$$

where $p(x, y, t)$ is the population number density at position $(x, y)$ and time $t$. The dispersal kernel $\phi\left(\Delta_{x}, \Delta_{y}\right)$ is the probability per unit area that a particle (or individual) that was at $\left(x+\Delta_{x}, y+\Delta_{y}, t\right)$ jumps to $(x, y, t+T) . T$ is the time interval between two subsequent jumps (usually $T=1$ generation $\left.^{10,11}\right)$. The last term, $R[p(x, y, t)]$, corresponds to the reaction (or reproduction) process. In contrast to, e.g., human populations, ${ }^{10,14}$ dispersal (of seeds) for tree populations takes place during a specific period of the year only, always after reproduction (seed production). The dispersal and reproduction are not independent, so a time-ordered evolution equation for the adult tree number density must be 
used, instead of Eq. (1). The time-ordered evolution equation is $^{16,17}$

$$
\begin{aligned}
p(x, y, t+T)= & R_{0} \int_{-\infty}^{+\infty} \int_{-\infty}^{+\infty} p\left(x+\Delta_{x}, y+\Delta_{y}, t\right) \\
& \times \phi\left(\Delta_{x}, \Delta_{y}\right) d \Delta_{x} d \Delta_{y}
\end{aligned}
$$

where $R_{0}$ is the net reproductive rate (number of seeds per parent tree and year which survive into an adult tree), and $T$ is the age (in yr) at first reproduction ( $T$ is sometimes called the generation time). Equation (2) is exact only for species with nonoverlapping generations (i.e., such that parents reproduce only once and then die). ${ }^{16,17}$ But previous $1 \mathrm{D}$ results with hypothetical kernels show that substantially more complicated, age-structured models do not change the order of magnitude of the front speed. ${ }^{18,19}$ Thus, the interesting case of age-structured extensions of the 2D Eq. (2) will be tackled in future work. Here we will use the approximate Eq. (2). This will make it much easier to focus our attention on the main physical question posed in the Introduction: How do the dispersion kernels in Ref. 7 affect the speed of fronts? The kernels in Ref. 7 have two components: a short-distance one, "S" (with probability $p_{S}$ and distances $\sim 10^{2} \mathrm{~m}$ ), and a long-distance one, "L" (covering distances of $10^{3}-10^{4} \mathrm{~m}$ but with probability $\left.p_{L}=1-p_{S} \ll p_{S}\right)$. The latter component is due to atmospheric turbulent-uplifting events, which have been up to now neglected in the prediction of invasion speeds. Both components are clearly distinguished in, e.g., Fig. 2 in Ref. 7 and they are due to independent events (because a seed can reach any final location either after being uplifted or not, but both possibilities are obviously incompatible). Therefore, the dispersion kernel can be written in the additive form $\phi\left(\Delta_{x}, \Delta_{y}\right)=p_{S} \phi_{S}\left(\Delta_{x}, \Delta_{y}\right)+p_{L} \phi_{L}\left(\Delta_{x}, \Delta_{y}\right)$.

\section{CONTINUOUS-SPACE RANDOM WALK (CSRW) MODEL}

For the evolution equation [Eq. (2)], assuming $R_{0}>1$ and that $p(x, y, t)$ has bounded support (i.e., vanishes outside a finite region) at some finite value of time, Weinberger presented a general approach to derive the front speed. ${ }^{16,17}$ But Weinberger's approach has been almost always applied to one dimension. ${ }^{18,19}$ Thus, it will be clarifying to deal briefly with the $2 \mathrm{D}$ case in this section. Also, we will use a much simpler approach than Weinberger's.

The speed of radially symmetric 2D front solutions can be found most easily by assuming that for $t \rightarrow \infty$, the front is approximately planar at scales much larger than that of individual dispersal events. We can then choose the $x$ axis parallel to the local velocity of the front. Let $c \equiv\left|c_{x}\right|$ stand for this speed $\left(c_{y}=0\right.$ in the local frame just introduced) ${ }^{8,23} \mathrm{We}$ look for constant-shape solutions with the form $p=p_{0} \exp$ $[-\lambda(x-c t)]$ as $x-c t \rightarrow \infty$ and, as usual, assume that the minimum speed is the one of the front ${ }^{8,9}$ (we will check this assumption by means of numerical simulations in Fig. 1). Then Eq. (2) leads to the asymptotic $(t \rightarrow \infty)$ speed of $2 \mathrm{D}$ fronts,

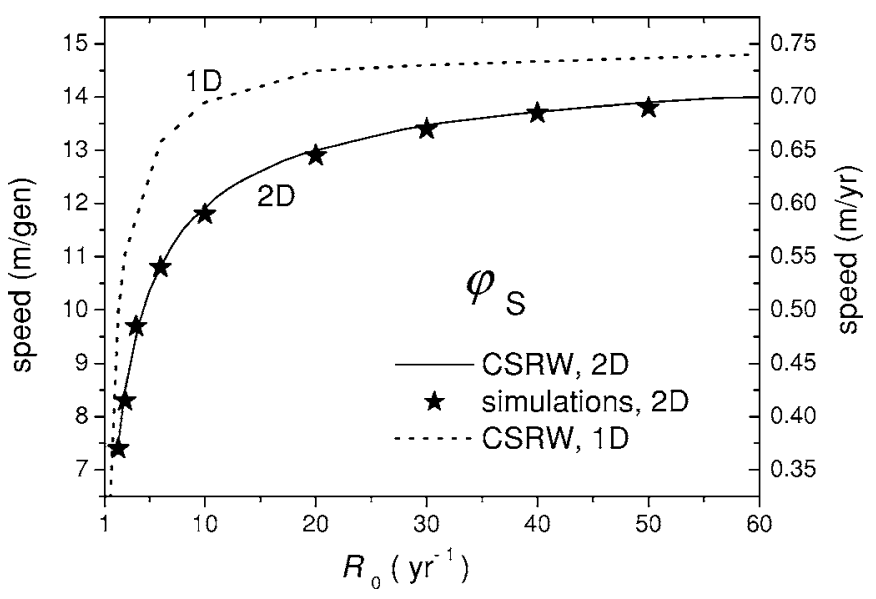

FIG. 1. Front speed in two dimensions vs net reproductive rate (Ref. 25) for a unimodal short-distance kernel $\varphi_{S}(\Delta)$. Stars: 2D computer simulations. Full curve: analytical 2D CSRWs, Eqs. (3)-(5). There is good agreement. The 1D speed for the same kernel is included for comparison (dotted curve). For bimodal kernels it is found that computer simulations cannot yield accurate results within a reasonable computing time (Sec. IV). The DSRW model overcomes this limitation (Fig. 2).

$$
c=\min _{\lambda>0} \frac{\ln \left[R_{0} \hat{\varphi}(\lambda)\right]}{\lambda T},
$$

where

$$
\hat{\varphi}(\lambda) \equiv \int_{0}^{\infty} d \Delta \varphi(\Delta) I_{0}(\lambda \Delta)
$$

and

$$
I_{0}(\lambda \Delta) \equiv \frac{1}{2 \pi} \int_{0}^{2 \pi} d \theta \exp (\lambda \Delta \cos \theta)
$$

is the modified Bessel function of the first kind and order zero. We have related the dispersal probability per unit area $\phi(\Delta)$ (i.e., into a rectangular area $d \Delta_{x} d \Delta_{y}$ ) to that per unit length $\varphi(\Delta)$ (i.e., into a 2D ring of area $2 \pi \Delta d \Delta$ ), because the kernel in Ref. 7 is $\varphi(\Delta)$. It is easy to see that

$$
\varphi(\Delta)=2 \pi \Delta \phi(\Delta) .
$$

We have assumed an isotropic dispersion kernel (i.e., that $\phi$ depends only on distance $\Delta \equiv \sqrt{\Delta_{x}^{2}+\Delta_{y}^{2}}$ ) and obviously

$$
\int_{0}^{\infty} d \Delta \varphi(\Delta)=1
$$

Previous papers on Reid's paradox ${ }^{18,19}$ usually apply the corresponding 1D result instead of (3)-(5). In fact, in one dimension, Eq. (3) holds but Eqs. (4) and (5) do not. Thus, speed $c$ is different in one dimension than in two dimensions [indeed, in Sec. VI we show that the 2D speed is always slower than in one dimension for the same dispersal kernel $\varphi(\Delta)]$.

The fact that $1 \mathrm{D}$ and $2 \mathrm{D}$ speeds are different was noted already in Ref. 22 for the evolution equation in (1) and in Ref. 10 for its hyperbolic and Fisher limits. However, here we will deal with the evolution equation in (2).

Since we are interested in Reid's paradox, which refers to forest range expansions that took place in two dimensions, 
we will firstly deal with the 2D case. We shall compare speeds in two dimensions and one dimension only in Sec. VI.

\section{MOLECULAR DYNAMICS SIMULATIONS}

We are not dealing with a differential but with an integrodifference equation in two dimensions, Eq. (2). Therefore, numerical simulations in this paper will not be based on finite-step approximations to derivatives but on molecular dynamics (or cellular automata in the continuous limit). Simulations in two dimensions are much more time costly than in one dimension, but a 2D model is necessary (as seen in the last paragraph of the previous section). We have thus performed simulations on a $2 \mathrm{D}$ grid, with nearest neighbors separated by a distance $D$. Initially $p(x, y, 0)=1$ at $(x, y)$ $=(0,0)$, and 0 elsewhere. At each time step, we compute the new number density of trees $p(x, y, t+T)$ at all nodes of the 2D grid as follows. In agreement with Eq. (2), we first compute the seed production $R_{0} p(x, y, t)$ at every node ${ }^{24}$ and then redistribute this value among all grid nodes using the kernel $\phi(\Delta)$. We have performed such 2D simulations for values of $R_{0}$ and $T$ typical of the yellow poplar (Liriodendron tulipifera). ${ }^{25}$ We consider this species because its long- and short-distance kernel components $\left[\varphi_{S}(\Delta)\right.$ and $\varphi_{L}(\Delta)$, respectively] were determined in Ref. 7. Consider first a very simple, short-distance unimodal kernel $\varphi_{S}(\Delta)$ such that it is approximately constant for dispersal distances $\Delta<15 \mathrm{~m}$ and zero for $\Delta>15 \mathrm{~m}^{7}$ Using a $2 \mathrm{D}$ grid with nearest neighbors separated by a distance $D=1 \mathrm{~m}$, the simulations agree with the CSRW, as shown in Fig. $1 .^{26}$ This shows (i) the validity of the minimum-speed conjecture ${ }^{27}$ and (ii) the need to take Eq. (6) into account in the simulations. ${ }^{28}$ But $\varphi_{S}(\Delta)$ is a unimodal kernel, whereas we are interested in bimodal ones (see Sec. I). Then the simulations above are not useful, as we have found that the time required is prohibitively long. ${ }^{29}$ Therefore, molecular dynamics simulations are not practical to test the 2D analytical result in (3) for bimodal kernels. Is there some other way to test whether Eq. (3) holds or not for bimodal kernels? In the next section we present a fast, efficient approach that we have found very useful for this purpose.

$$
c=\min _{\lambda>0} \frac{\ln \left(R_{0}\{P(D)[\cosh (\lambda D)+1] / 2+P(D \sqrt{2}) \cosh (\lambda D)\}\right)}{\lambda T} .
$$

For the simple case of Eq. (8), $n=2$ and the only possible dispersal distances are $\Delta_{1}=D$ and $\Delta_{2}=D \sqrt{2}$.

We apply the approach already used above [Eq. (3)] (Refs. 8, 23, and 27) and obtain, from the DSRW equation [Eq. (8)],

\section{DISCRETE-SPACE RANDOM WALK (DSRW) MODEL}

This model is not exact, but it is necessary to check the CSRW model for bimodal kernels. The DSRW is closely analogous to the numerical simulations, in the following sense. Both in the DSRW and the simulations, we replace 2D continuous space by a grid of points (nodes) with nearest neighbors separated by a distance $D$ along the $x$ and $y$ axes. The nodes are the only points available for seeds and trees. First consider the very simple, highly idealized case in which any tree disperses seeds only to its eight nearest neighbors on the grid. Obviously, these eight final dispersal nodes lie on a square with side $2 D$ and center at the parent tree, as follows. The closest four nodes are a distance $\pm D$ away along the $x$ or $y$ direction, and the next four are at distance $\pm D$ away along both directions, i.e., on the vertices of the square (at distance $\sqrt{D^{2}+D^{2}}=D \sqrt{2}$ from the parent tree). Then Eq. (2) becomes simply

$$
\begin{aligned}
p(x, y, t+T)= & R_{0}\{[P(D) / 4][p(x-D, y, t)+p(x+D, y, t) \\
& +p(x, y-D, t)+p(x, y+D, t)] \\
& +[P(D \sqrt{2}) / 4][p(x-D, y-D, t) \\
& +p(x-D, y+D, t)+p(x+D, y-D, t) \\
& +p(x+D, y+D, t)]\}
\end{aligned}
$$

where the first four terms correspond to horizontal and vertical "jumps," whereas the last four terms are due to diagonal jumps, and the jump probabilities are, from Eq. (6),

$$
P\left(\Delta_{i}\right)=\frac{\phi\left(\Delta_{i}\right)}{\sum_{j=1}^{n} \phi\left(\Delta_{j}\right)}=\frac{\varphi\left(\Delta_{i}\right) /\left(2 \pi \Delta_{i}\right)}{\sum_{j} \varphi\left(\Delta_{j}\right) /\left(2 \pi \Delta_{j}\right)} .
$$

Note that Eq. (10) is a very simple approximation (DSRW) but is completely analogous to the exact (CSRW) speed in (3). We have found [e.g., for $\varphi_{S}(\Delta)$ in Sec. IV] that this extremely simple DSRW yields a speed in (10) which disagrees with that from the CSRW. Thus, we next consider dispersal to nodes not on a single but on many squares $(j=1,2,3, \ldots)$ centered at each parent tree. A square with side $2 j D$ will obviously have $8 j$ nodes, namely, four at dis- tance $j D$, four at distance $j D \sqrt{2}$, and also (except for the simple case $j=1$ above) eight nodes at distance $\sqrt{(j D)^{2}+(i D)^{2}}$ for $i=1,2, \ldots, j-1$. Finally, in order to use the measured kernels ${ }^{7}$ we need to restrict dispersal to a maximum distance in whatever direction, $r_{\max }$. Then it is not difficult to write the analog to Eq. (8) for a bimodal kernel [i.e., $\left.p_{L} \varphi_{L}(r)+p_{S} \varphi_{S}(r)\right]$ and see that the speed in (10) is generalized into 


$$
\begin{aligned}
c= & \min _{\lambda>0} \frac{1}{\lambda_{T}} \ln \left[R _ { 0 } p _ { L } \sum _ { j = 0 } ^ { N _ { L } } \left(P_{L}\left(j D_{L}\right) \frac{\left[\cosh \left(\lambda j D_{L}\right)+1\right]}{2}+P_{L}\left(j D_{L} \sqrt{2}\right) \cosh \left(\lambda_{j} D_{L}\right)\right.\right. \\
& \left.+\sum_{i=1}^{j-1}\left\{P_{L}\left[\sqrt{\left(j D_{L}\right)^{2}+\left(i D_{L}\right)^{2}}\right] \frac{\cosh \left(\lambda i D_{L}\right)+\cosh \left(\lambda j D_{L}\right)}{2}\right\}\right) \\
& +p_{S} \sum_{j=0}^{N_{S}}\left(P_{S}\left(j D_{S}\right) \frac{\left[\cosh \left(\lambda j D_{S}\right)+1\right]}{2}+P_{S}\left(j D_{S} \sqrt{2}\right) \cosh \left(\lambda j D_{S}\right)\right. \\
& \left.\left.+\sum_{i=1}^{j-1}\left\{P_{S}\left[\sqrt{\left(j D_{S}\right)^{2}+\left(i D_{S}\right)^{2}}\right] \frac{\cosh \left(\lambda i D_{S}\right)+\cosh \left(\lambda j D_{S}\right)}{2}\right\}\right)\right],
\end{aligned}
$$

where $N_{L}=r_{\max L} / D_{L}, N_{S}=r_{\max S} / D_{S}$, and the terms with $\sqrt{\left(j D_{S}\right)^{2}+\left(i D_{S}\right)^{2}}$ arise from jumps in directions different from $0^{\circ}$, $\pm 45^{\circ}, 180^{\circ}$, and $\pm 90^{\circ}$. The probabilities are related by Eq. (9) to the corresponding dispersion kernel, for example,

$$
P_{L}\left(j D_{L}\right)=\frac{\varphi_{L}\left(j D_{L}\right) / 2 \pi j D_{L}}{\sum_{j}^{N_{L}}\left\{\varphi_{L}\left(j D_{L}\right) / 2 \pi j D_{L}+\varphi_{L}\left(j D_{L} \sqrt{2}\right) / 2 \pi j D_{L} \sqrt{2}+\sum_{i=1}^{j-1} \varphi_{L}\left[\sqrt{\left(j D_{S}\right)^{2}+\left(i D_{S}\right)^{2}}\right] / 2 \pi \sqrt{\left(j D_{S}\right)^{2}+\left(i D_{S}\right)^{2}}\right\}} .
$$

For the yellow poplar (Liriodendron tulipifera), the LDD component of the kernel derived (and favorably compared to observations) in Refs. 7, 20, and 21 can be fitted to a curve of the form

$$
\varphi_{L}(\Delta)= \begin{cases}0, & \Delta<10^{3} \mathrm{~m} \\ 10^{3.903} \Delta^{-2.301}, & 10^{3} \mathrm{~m} \leqslant \Delta \leqslant 10^{4} \mathrm{~m} \\ 0, & \Delta>10^{4} \mathrm{~m},\end{cases}
$$

so $r_{\max L}=10^{4} \mathrm{~m}$, whereas, as mentioned in Sec. IV, its shortdistance component $\varphi_{S}(\Delta)$ can be taken as approximately constant for $\Delta<15 \mathrm{~m}$ and zero for $\Delta>15 \mathrm{~m}$, so $r_{\max s}$ $=15 \mathrm{~m}$.

Figure 2 presents the results for the bimodal kernel $p_{L} \varphi_{L}(r)+p_{S} \varphi_{S}(r)$, where $p_{L}=0.00202$ and $p_{S}=1-p_{L}$ are the probabilities of long-distance and short-distance dispersals (obtained from Fig. 2 in Ref. 7). The results for the unimodal kernels $\varphi_{L}(r)$ and $\varphi_{S}(r)$ are also presented for comparison. The relevance of these results for Reid's paradox will be discussed in Sec. VII.

\section{COMPARISON OF INVASION SPEEDS IN TWO DIMENSIONS AND ONE DIMENSION}

The aim of this paper has been to analyze front speeds in 2D space. But many papers have applied 1D results instead, see, e.g., Refs. 18, 19, 30, and 31 and references therein. Thus, a comparison between two dimensions and one dimension is appropriate.

In one dimension, clearly the kernel per unit area $\phi(\Delta)$ has no physical meaning. The $1 \mathrm{D}$ evolution equation equivalent to Eq. (2) is obviously

$$
p(x, t+T)=R_{0} \int_{-\infty}^{+\infty} p\left(x+\Delta_{x}, t\right) \widetilde{\varphi}\left(\Delta_{x}\right)
$$

where we have not used the notation $\varphi(\Delta)$ for the kernel in order to avoid confusion. Indeed, in two dimensions we have used Eq. (7) for the normalization of the kernel per unit length $\varphi$,

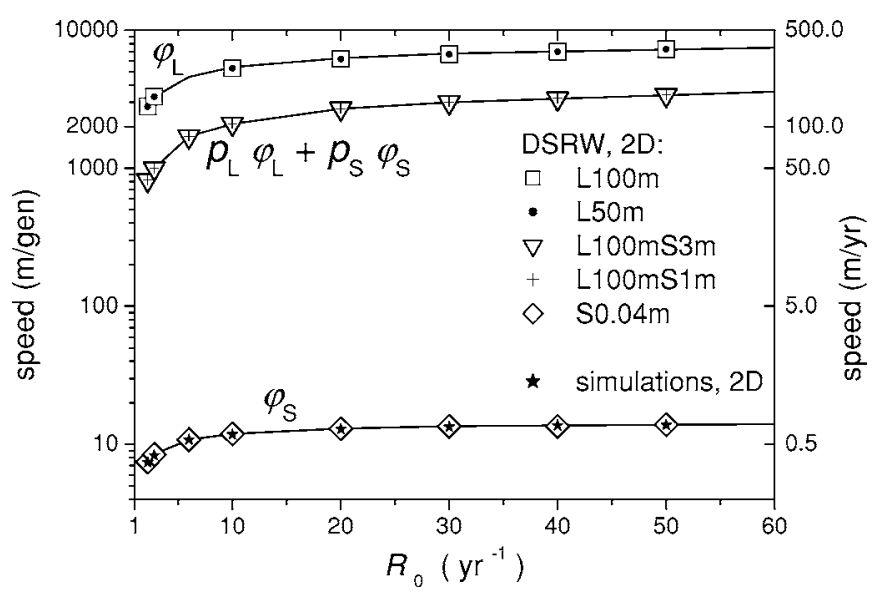

FIG. 2. Front speeds in two dimensions vs net reproductive rate (Ref. 25). Curves: CSRWs, Eqs. (3)-(5). Symbols: DSRWs, Eq. (11), using the values of $D_{L}$ and/or $D_{S}$ in the legend (in meters) and the corresponding kernel(s). The bimodal kernel for the yellow poplar, from Ref. 7, leads to the middle curve. It thus predicts speeds of about $10^{2}-10^{3} \mathrm{~m} /$ generation. In contrast, the short-range unimodal kernel (lower curve and stars, the same as in Fig. 1) predicts front speeds several orders of magnitude lower. Thus, the bimodal kernels derived and favorably compared to data in Refs. 7, 20, and 21 may solve Reid's paradox. Note from the upper curve (100\% of seeds with LDD,$\left.\varphi_{L}\right)$ that the same order of magnitude $\left(10^{2} \mathrm{~m} / \mathrm{yr}\right)$ is obtained for only $0.2 \%$ of seeds with LDD (middle curve). 


$$
\int_{0}^{\infty} d \Delta \varphi(\Delta)=1
$$

In contrast, in the $1 \mathrm{D} \mathrm{Eq.} \mathrm{(14)} \mathrm{the} \mathrm{kernel} \mathrm{per} \mathrm{unit} \mathrm{length} \tilde{\varphi}$ is defined both for positive and negative jumps. Therefore, the lower integration limit must now be $-\infty$ instead of 0 , i.e.,

$$
\int_{-\infty}^{\infty} d \Delta_{x} \widetilde{\varphi}\left(\Delta_{x}\right)=1
$$

Clearly, $\Delta_{x}$ can be either positive or negative, whereas $\Delta$ $\geqslant 0$ (they are related as $\Delta \equiv\left|\Delta_{x}\right|$ in one dimension and $\Delta$ $\equiv \sqrt{\Delta_{x}^{2}+\Delta_{y}^{2}}$ in two dimensions).

In order to compare to two dimensions, note that for an isotropic kernel, Eq. (16) implies that

$$
\int_{0}^{\infty} d \Delta_{x} \tilde{\varphi}(\Delta)=\frac{1}{2}
$$

If we introduce the following 1D kernel (which we define only for $\Delta \equiv\left|\Delta_{x}\right| \geqslant 0$ ):

$$
\varphi(\Delta)=2 \widetilde{\varphi}(\Delta), \quad \text { if } \Delta \geqslant 0
$$

then Eq. (17) yields

$$
\int_{0}^{\infty} d \Delta \varphi(\Delta)=1
$$

which agrees with the corresponding normalization equation in two dimensions, see Eq. (15). This will make it possible to perform a meaningful comparison between one dimension and two dimensions.

Now that we have carefully defined the kernel per unit length $\varphi(\Delta)$ both in one dimension and two dimensions, we can ask the following question. For a given kernel $\varphi(\Delta)$, is the front in one dimension faster or slower than in two dimensions? Up to now, this question seems to have remained unanswered for arbitrary kernels $\varphi(\Delta)$. Here, we will provide a mathematical proof that the front is always slower in two dimensions than in one dimension for the same kernel $\varphi(\Delta)$.

For an isotropic 1D kernel, i.e., $\widetilde{\varphi}\left(-\Delta_{x}\right)=\widetilde{\varphi}\left(\Delta_{x}\right)$, Eq. (3) holds for the front speed $c$ but $\hat{\varphi}(\lambda)$ is not given by the 2D Eq. (4). Instead, in one dimension

$$
\hat{\varphi}(\lambda)=\int_{-\infty}^{\infty} d \Delta_{x} \tilde{\varphi}\left(\Delta_{x}\right) \exp \left(\lambda \Delta_{x}\right) .
$$

This result was first derived by Weinberger ${ }^{16,17}$ and has been widely applied $^{18,19,30,31}$ (it can be also easily obtained from the approach in Sec. III above).

The 1D speed can thus be written in terms of $\varphi(\Delta)$, from Eqs. (20) and (18),

$$
c_{1 \mathrm{D}}=\min _{\lambda>0} \frac{\ln \left\{R_{0} \int_{0}^{\infty} d \Delta \varphi(\Delta)(1 / 2)[\exp (-\lambda \Delta)+\exp (\lambda \Delta)]\right\}}{\lambda T},
$$

The 2D speed for an isotropic kernel $\varphi(\Delta)$ is, from Eqs. (3)-(5),

$$
c_{2 \mathrm{D}}=\min _{\lambda>0} \frac{\ln \left\{R_{0} \int_{0}^{\infty} d \Delta \varphi(\Delta)(1 / 2 \pi) \int_{0}^{\pi} d \theta[\exp (-\lambda \Delta \cos \theta)+\exp (\lambda \Delta \cos \theta)]\right\}}{\lambda T},
$$

where we have simply applied that $\cos (\alpha+\pi)=-\cos \alpha$. Alternatively, Eq. (22) can be also obtained from Eq. (24.102) in Ref. 32. Finally, we write the $2 \mathrm{D}$ speed in $(22)$ as

$$
c_{2 \mathrm{D}}=\min _{\lambda>0} \frac{\ln \left[R_{0} \int_{0}^{\infty} d \Delta \varphi(\Delta)(1 / \pi) \int_{0}^{\pi} d \theta \cosh (\lambda \Delta \cos \theta)\right]}{\lambda T},
$$

where we have simply used the definition $\cosh x \equiv \frac{1}{2}[\exp (x)$ $+\exp (-x)]$.

We now rewrite the $1 \mathrm{D}$ speed in (21) in a form similar to the 2D speed in (22). Obviously,

$$
c_{1 \mathrm{D}}=\min _{\lambda>0} \frac{\ln \left[R_{0} \int_{0}^{\infty} d \Delta \varphi(\Delta)(1 / \pi) \int_{0}^{\pi} d \theta \cosh (\lambda \Delta)\right]}{\lambda T} .
$$

Comparing Eqs. (23) and (24) it is obvious that, for any symmetric kernel per unit length $\varphi(\Delta)$ (defined for $\Delta \geqslant 0$ ),

$$
c_{2 \mathrm{D}}<c_{1 \mathrm{D}}
$$

simply because $\cos \theta \leqslant 1$ and $\cosh x \equiv \frac{1}{2}[\exp (x)+\exp (-x)]$ increases with increasing values of $x \geqslant 0$ (in other words, its derivative is $\sinh x \equiv \frac{1}{2}[\exp (x)-\exp (-x)] \geqslant 0$ if $\left.x \geqslant 0\right)$.

Therefore, we have shown that all symmetric kernels 
$\varphi(\Delta)$ give a speed which is lower in two dimensions than in one dimension. This result is not surprising, according to the following two arguments.

(i) We may understand intuitively the result in (25) as follows. Consider, for example, a delta kernel, i.e., such that all individuals move the same distance. In one dimension, half of them will travel in each direction. In two dimensions, fewer individuals can reach a given point than in one dimension because there are not only two final dispersal points but a whole a circle. So, we could well expect the front to be slower in two dimensions than in one dimension. Moreover, any kernel can be regarded as a sum of delta kernels. From this perspective, it is not surprising that fronts are slower in two dimensions than in one dimension.

(ii) For the special cases of Fisher and hyperbolic reaction-diffusion evolution equations, fronts are also slower in two dimensions than in one dimension. ${ }^{10}$ However, it must be remembered that (i) such approaches are diffusive limits to Eq. (1), which does not take proper care of the time order of events [in contrast to Eq. (2) or (14), considered here], and (ii) diffusive limits are well known to break down for long-distance dispersal. ${ }^{18}$

All of the results in the present paper, including Eq. (25), have been obtained for the time-ordered evolution equations (2) in two dimensions and (14) in one dimension. Thus, they may not hold for other evolution equations [such as (1)].

In this section we have shown that, for time-ordered evolution dynamics and, an arbitrary symmetric kernel per unit length $\varphi(\Delta)$, the front is always slower in two dimensions than in one dimension. ${ }^{33}$

For the short-distance kernel $\varphi_{S}(r)$, we include the predicted speed in (21) in one dimensional as a dotted curve in Fig. 1. It is seen that the $1 \mathrm{D}$ speed is faster than the $2 \mathrm{D}$ speed, as it should. If the 1D approach is applied, the error (difference relative to the 2D prediction) is important and increases rapidly for decreasing values of $R_{0}$ (for example, the error is $22 \%$ for $R_{0}=6 \mathrm{yr}^{-1}$ and $33 \%$ for $R_{0}=2.2 \mathrm{yr}^{-1}$ ). This could have been expected since the lower the net reproductive rate, the more important is dispersal relative to reproduction. The differences between the $2 \mathrm{D}$ and $1 \mathrm{D}$ results show the importance of using 2D formulas to predict invasion speeds. Similarly, for the bimodal kernel $p_{L} \varphi_{L}(r)$ $+p_{S} \varphi_{S}(r)$, Fig. 3 shows the predicted speed in (21) in one dimension as a dotted curve. Again, it is faster than in two dimensions, as it should, and the differences are important, so it is not reasonable to use $1 \mathrm{D}$ models for invasions that take place in two dimensions (e.g., postglacial forest range expansions).

\section{DISCUSSION}

Here, molecular dynamics simulations have been useful to (i) make us realize that $\phi(r)$ must be related to the measured kernel $\varphi(r)$ (Ref. 28) and (ii) check that the minimum speed in (11) is that of the front. ${ }^{27}$ Thus, it is not reasonable to rely on a single analytic approach only (e.g., the CSRW).

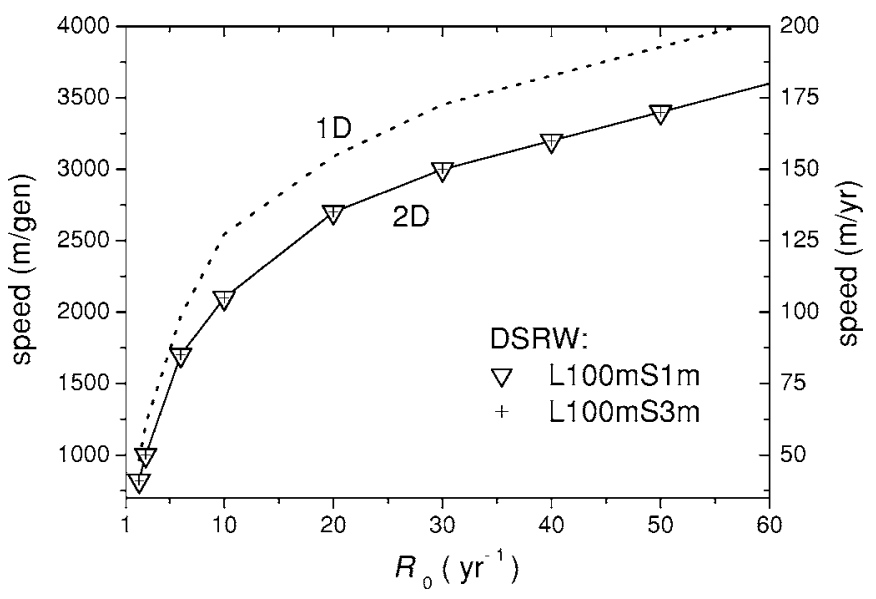

FIG. 3. Comparison of speeds in two dimensions and one dimension for the bimodal kernel. The full line and symbols correspond to two-dimensions (the same as in Fig. 2), whereas the dotted curve corresponds to one dimension. The $1 \mathrm{D}$ speed is about $20 \%$ faster than the $2 \mathrm{D}$ speed.

Indeed, in reaction-diffusion problems, simulations are almost always used to check the analytic results. ${ }^{8,9}$ However, for bimodal kernels the 2D simulations do not yield accurate enough results within a viable computing time (Sec. IV). Thus, in addition to the 2D CSRW model, we have also presented a discrete analytical model (DSRW) and applied it (instead of the simulations) to check the CSRW results (Figs. 2 and 3 ).

Both the 2D DSRW and 2D CSRW models show conclusively that the front speeds for the bimodal kernels in Ref. 7 [i.e., $p_{L} \varphi_{L}(r)+p_{S} \varphi_{S}(r)$ ] are about $10^{2}-10^{3} \mathrm{~m} / \mathrm{yr}$, i.e., two orders of magnitude faster than those for the unimodal, short-range component $\varphi_{S}(r)$ (Fig. 2). Speeds of $10^{2}-10^{3} \mathrm{~m} / \mathrm{yr}$ are, in fact, those required to solve Reid's paradox. $^{18}$

Short-distance kernels $\varphi_{S}(r)$ have been measured experimentally many times. But bimodal kernels with a LDD component $\varphi_{L}(r)$ were derived by a mechanistic (or physical) model in Ref. 7 which motivated the work here reported.

We conclude that Reid's paradox of rapid tree migration can be solved (as far as the order of magnitude is concerned) by taking into account the bimodal dispersal kernels derived and favorably compared to data in Refs. 7, 20, and 21. This is the main biophysical result of this work.

On the analytical side, our main contributions here are the DSRW model, taking into account two dimensions in the CSRW, and the comparison between two dimensions and one dimension (Sec. VI). But the important natural phenomenon tackled is Reid's paradox, which motivated all of our analytical work and 2D simulations. Of course, we do not claim to have solved Reid's paradox. In future work, it would be interesting to apply the 2D CSRW and DSRW approaches presented here by comparing the predictions and observations for a list of tree species such that (i) their range expansion speeds can be measured from the fossil record and (ii) dispersal kernels and net reproductive rates can be estimated using the methods in Ref. 7 and experimental measurements. Our results here do show the procedures (2D CSRWs and 2D DSRWs) useful to perform such an analysis (as well as the limitations of using numerical simulations for this purpose). 
Section VI contains the proof that, for an arbitrary kernel $\varphi(\Delta)$, the front is always slower in two dimensions than in one dimension under the time-ordered evolution equation (2) (in two dimensions) or Eq. (14) (in one dimension).

It future work, it would be nice to apply the physical models presented (2D CSRWs and 2D DSRWs) to extended 2D evolution equations that take into account age structure, interspecific interactions, etc. It would be also interesting to generalize the models described here to a variety of applied biophysics systems, e.g., tumor growth models ${ }^{1}$ with several nutrients (each with a different diffusion coefficient or dispersal kernel), several-species dispersion in microchannel flows, ${ }^{2}$ etc.

\section{ACKNOWLEDGMENTS}

Funded by European Commission Grant No. NEST28192-FEPRE, MEC-FEDER Grant No. FIS-2006-12296C02-02, and Generalitat de Catalunya Grant No. SGR-200500087 .

${ }^{1}$ A. L. Garner, Y. Y. Lau, T. L. Jackson, M. D. Uhler, D. W. Jordan, and R. M. Gilgenbach, J. Appl. Phys. 98, 124701 (2005).

${ }^{2}$ R. F. Ismagilov, A. D. Stroock, P. J. A. Kenis, G. Whitesides, and H. A. Stone, Appl. Phys. Lett. 76, 2376 (2000).

${ }^{3}$ P. R. Nair and M. A. Alam, Appl. Phys. Lett. 88, 233120 (2006)

${ }^{4}$ G. Chen, Phys. Rev. Lett. 86, 2297 (2001).

${ }^{5}$ D. B. Olfe, AIAA J. 5, 638 (1967).

${ }^{6}$ M. F. Modest, Radiative Heat Transfer (McGraw-Hill, New York, 1993).

${ }^{7}$ R. Nathan, G. G. Katul, H. S. Horn, S. M. Thomas, R. Oren, R. Avissar, S.

W. Pacala, and S. A. Levin, Nature (London) 418, 409 (2002).

${ }^{8}$ J. Fort and V. Méndez, Rep. Prog. Phys. 65, 895 (2002).

${ }^{9}$ W. van Saarloos, Phys. Rep. 386, 29 (2003).

${ }^{10}$ J. Fort and V. Méndez, Phys. Rev. Lett. 82, 867 (1999).

${ }^{11}$ J. Fort and V. Méndez, Phys. Rev. Lett. 89, 178101 (2002).

${ }^{12}$ K. Davison, P. Dolukhanov, G. R. Sarson, and A. Shukurov, J. Archaeol. Sci. 33, 641 (2006).

${ }^{13}$ M. O. Vlad and J. Ross, Phys. Rev. E 66, 061908 (2002).

${ }^{14}$ J. Fort, D. Jana, and J. M. Humet, Phys. Rev. E 70, 031913 (2004).

${ }^{15}$ J. Fort, J. Pérez, E. Ubeda, and J. García, Phys. Rev. E 73, 021907 (2006).

${ }^{16}$ H. F. Weinberger, in Nonlinear Partial Differential Equations and Applications, edited by J. Chadam (Springer, Berlin, 1978).

${ }^{17}$ H. F. Wienberger, SIAM J. Math. Anal. 13, 353 (1982).

${ }^{18}$ J. S. Clark, Am. Nat. 152, 204 (1998) (especially Fig. 1 and p. 219).

${ }^{19}$ M. Neubert and H. Caswell, Ecology 81, 1613 (2000) (especially Fig. 15).

${ }^{20}$ R. Nathan and G. G. Katul, Proc. Natl. Acad. Sci. U.S.A. 102, 8251
(2005).

${ }^{21}$ M. B. Soons, G. W. Heil, R. Nathan, and G. G. Katul, Ecology 85, 3056 (2004).

${ }^{22}$ V. Méndez, D. Campos, and J. Fort, Europhys. Lett. 66, 902 (2004).

${ }^{23}$ J. Fort and V. Méndez, Phys. Rev. E 60, 5894 (1999).

${ }^{24}$ Note that Eq. (2) does not include saturation. We have checked that including saturation in the $2 \mathrm{D}$ simulations, the front speeds are the same. Obviously the same happens in the CSRW and DSRW approaches after linearization.

${ }^{25}$ According to field observations in sites close to those where the dispersal kernel was measured, the fecundity $f$ of this species is of the order of $10^{4}$ seeds dispersed/tree yr, and its postdispersal seed-to-adult survival probability is $s \simeq 0.06 \%$ (see Ref. 7 and Refs. 25 and 26 therein). Thus we estimate the net reproductive rate, $R_{0}=f s$, to be in the range of 6-60 seeds/tree yr. The age at first reproduction (generation time) of the same species is $T \simeq 20 \mathrm{yr}$ (Ref. 18, Table 1).

${ }^{26}$ The numerical simulations agree with Eq. (2) but not with Eq. (1) with, e.g., $R[p(x, y, t)]=R_{0} p(x, y, t)$.

${ }^{27}$ The minimum speed [Eqs. (3)-(5) for the CSRW; Eq. (11) for the DSRW] is that of the front: this is seen by comparing to simulations (full curve in Fig. 1 for the CSRW; rhombs in Fig. 2 for the DSRW).

${ }^{28}$ The CSRW uses $\varphi(\Delta)$, which is the kernel found in Ref. 7. But the simulations and DSRW use $\phi(\Delta)$. Otherwise the agreement in Figs. 1 and 2 cannot be attained. For the DSRW, see especially Eq. (9).

${ }^{29}$ Several days of computing time are not enough for the bimodal kernel; in contrast, for the kernel $\varphi_{S}(\Delta)$ the necessary computing time is about $30 \mathrm{~min}$ (these results have been obtained for a virtual grid of $1000^{2}$ nodes and using a personal computer with an Intel Pentium III, 393 kbyte RAM and $1133 \mathrm{MHz}$ ).

${ }^{30}$ J. A. J. Metz, D. Mollison, and F. Van Den Bosch, The Geometry of Ecological Interactions, edited by V. Dieckmann, R. Law, and J. A. J. Metz (Cambridge University Press, Cambridge, 2000).

${ }^{31}$ M. Kot, M. A. Lewis, and P. van den Driessche, Ecology 77, 2027 (1996).

${ }^{32}$ M. R. Spiegel, Mathematical Handbook (McGraw-Hill, New York, 1970).

${ }^{33}$ For histogram data, in Ref. 34 it has been argued that $c_{1 \mathrm{D}}<c_{2 \mathrm{D}}$ for some specific kernels and parameter values. However, negative-displacement jumps $(\Delta x<0)$ in one dimension were apparently omitted [because Eq. (12) instead of (13) in Ref. 34 was apparently used to compute the 1D speeds in Table 1 there]. As the general proof in Sec. VI shows, one will always find that $c_{2 \mathrm{D}}<c_{1 \mathrm{D}}$, provided of course that jumps with $\Delta x<0$ are taken into account in one dimension. For nonhistogram data, the authors of Ref. 34 report that $c_{2 \mathrm{D}}<c_{1 \mathrm{D}}$ for some specific kernels and parameter values, in agreement with the general proof in Sec. VI in the present paper [but also in this case, their Eq. (9) instead of (8) in Ref. 34 should have been applied, again to take into account jumps with $\left.\Delta_{x}<0\right]$.

${ }^{34}$ M. A. Lewis, M. G. Neubert, H. Caswell, J. S. Clark, and K. Shea, in Conceptual Ecology and Invasion Biology: Reciprocal Approaches to Nature, edited by M. Cadotte, S. McMahon, and T. Fukami (Kluwer, Dordrecht, 2006), pp. 169-192. 